\title{
Improvement of Bilateral Elevated Foveal Retinoschisis Following Sequential Vitrectomy with ILM Peel in a Patient with Enhanced S Cone Syndrome
}

\section{Bedda $\mathrm{AM}^{*}$ and Amin RM}

Alexandria Faculty of Medicine, Ophthalmology department, Alexandria, Egypt

*Corresponding author: Bedda $\mathrm{AM}, \mathrm{MD}, \mathrm{PhD}$, Alexandria Faculty of Medicine, 6 Chamblion Street, Elazareeta Alexandria, Egypt, Tel: 00201222188091,E-mail: abedda@gmail.com.

Citation: Bedda AM, Amin RM (2017) Improvement of Bilateral Elevated Foveal Retinoschisis Following Sequential Vitrectomy with ILM Peel in a Patient with Enhanced S Cone Syndrome. J Case Rep Stud 5(3): 305. doi: 10.15744/2348-9820.5.304

Received Date: August 14, 2016 Accepted Date: June 28, 2017 Published Date: June 30, 2017

\begin{abstract}
Purpose: To report outcomes after vitrectomy with ILM peel for bilateral elevated foveoschisis in a patient with Goldmann-Favre syndrome.

Methods: Vitrectomy with ILM peel was done for one then the other eye of a 19 year-old patient with bilateral progressive enhanced s-cone syndrome associated elevated macular retinoschesis.

Results: Significant anatomical as well as visual improvements were noted in both eyes with regression of the macular edema, flattening of the retinoschesis, and a stable visual outcome thereafter.

Conclusion: Despite the inherent risks of surgery, vitrectomy with ILM peel may provide a possible surgical treatment option for patients with elevated retinal foveoschisis associated with enhanced S-cone syndrome.
\end{abstract}

Keywords: Enhanced S Cone Syndrome; Macular Cysts; Retinoschesis; Vitrectomy

\section{Introduction}

Enhanced S-cone syndrome is a bilateral hereditary vitreoretinal degenerative disorder characterized by decreased vision, early onset night blindness, vitreous liquefaction, peripheral pigmentary retinopathy, central or peripheral retinoschisis, and macular cystoid changes [1,2]. In many of those hereditary retinal dystrophies, cystic macular changes contribute to impaired vision, for which the underlying etiopathogenesis still remains unclear. While effective treatment is still lacking, some reports have shown carbonic anhydrase inhibitors to be potentially efficacious in treating some of these patients, including those with enhanced S-cone syndrome [3-5]. Our report, to our knowledge, is the first to describe anatomical as well as visual improvement after vitrectomy with internal limiting membrane (ILM) peel in one then the other eye of a patient with enhanced s-cone syndrome associated elevated macular retinoschesis with regression of the macular edema, flattening of the retinoschesis, and a stable visual outcome thereafter.

\section{Case Presentation}

A 19 year-old girl with enhanced S-cone syndrome presented with a recent further drop of vision in her right eye. She had had complaints of bilateral night blindness and diminished vision 9 years prior. A sibling; her brother, was reported to have had the same complaints.

At presentation, her best corrected visual acuity (BCVA) was 3/60 and 6/60 in the right and left eyes respectively. She had a normal anterior segment slit lamp exam with an intraocular pressure of 16 and $18 \mathrm{mmHg}$. Bilateral macular cystoid changes were noted on ophthalmoscopy with a markedly elevated retinoschisis in the right eye and peripheral pigmentary retinopathy (Figure 1A and B).

She had not received any specific treatment for her ocular condition over the preceding 9 years. Spectral domain optical coherence tomography (SD-OCT) demonstrated confluent macular cystoid changes in both eyes, with a severe elevated foveoschisis in the right eye. Foveal thickness was 1129 microns in the right eye and 239 microns in the left eye (Figure 1C and D). 


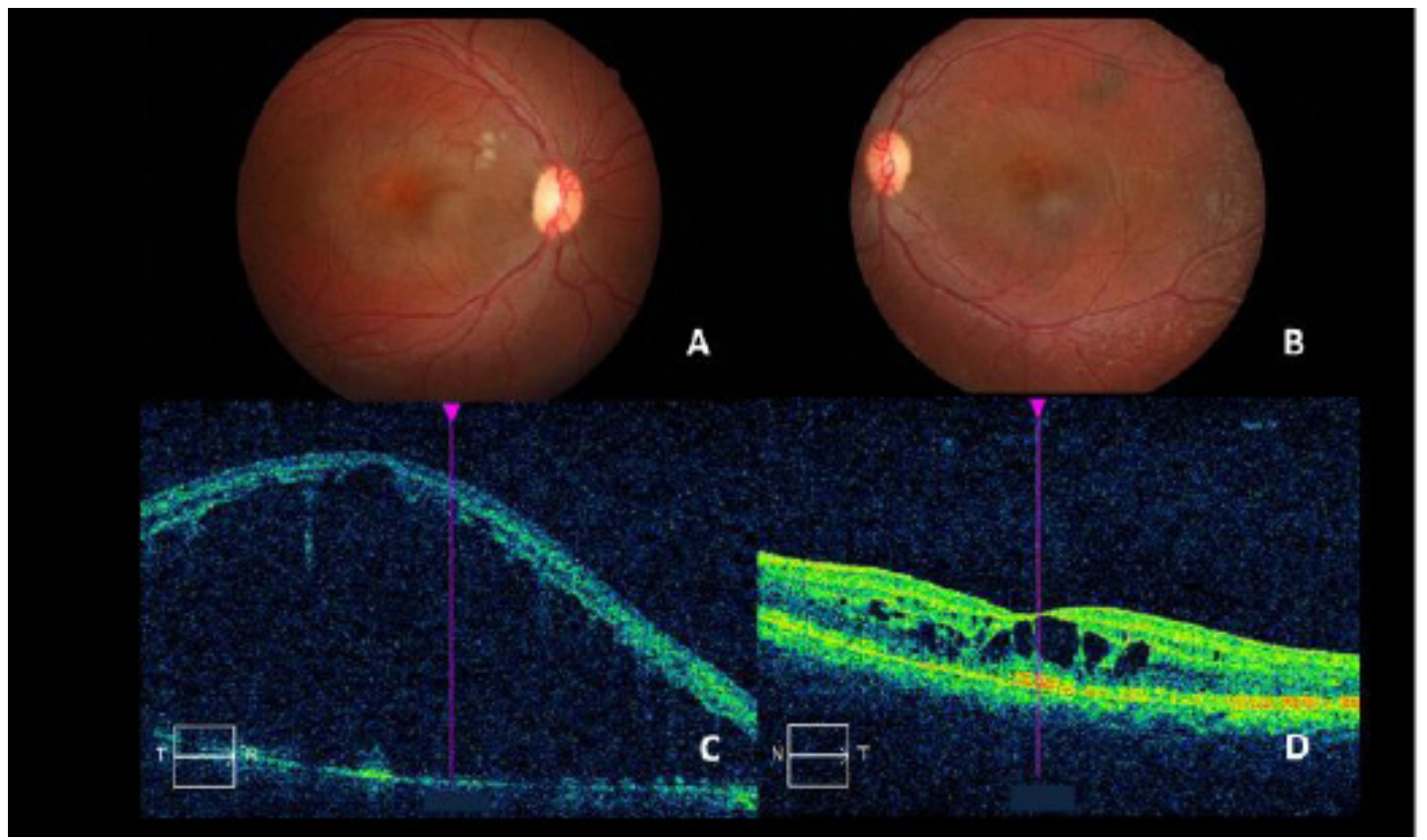

Figure 1: (A and B) Color fundus photographs of a patient with Goldmann-Favre syndrome with associated macular cystoid changes and elevated macular retinoschisis in the right eye; (C and D) SD-OCT scans demonstrating confluent macular cystoid changes in both eyes, with a severe elevated foveoschesis in the right eye. Foveal thickness was 1129 microns in the right eye and 239 microns in the left eye

Given the severity of the schisis, the patient was offered surgical intervention to help resolve her problem, to which the patient consented. She underwent a 23 gauge vitrectomy to the right eye with a Brilliant blue G (0.5\%) assisted ILM peel up to the arcades. $25 \%$ sulfur hexafluoride $\left(\mathrm{SF}_{6}\right.$ ) gas was used as postoperative gas tamponade with face-down position for 3 days. Two months following vitrectomy, the mean thickness of the central $1 \mathrm{~mm}$ foveal subfield of the right eye decreased from $1129 \mu \mathrm{m}$ to $201 \mu \mathrm{m}$. BCVA improved from 3/60 at presentation to 6/36 in the right eye (Figure 2).

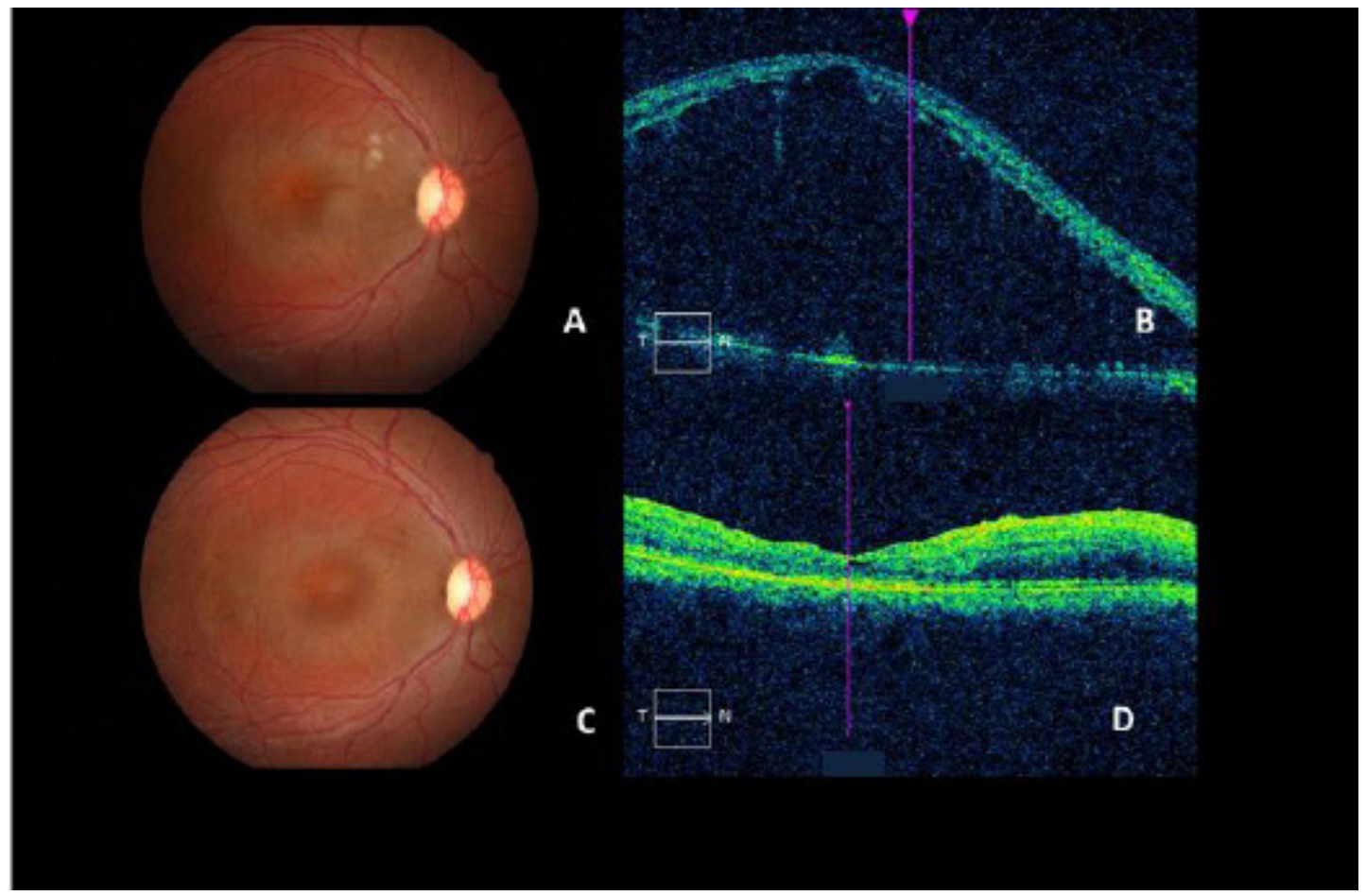

Figure 2: (A-D) Color fundus photographs and SD-OCT scans of the right eye of the patient with Goldmann-Favre syndrome two months after surgery showing complete flattening of the retinoschisis and resolution of the macular cystoid changes

Two years later, the patient returns with a stable vision of 6/36 in the right eye, and a drop of vision in her left eye from 6/60 down to 2/60. A marked elevation in the inner layer of the retina over the posterior pole is noted and SD-OCT shows a central thickness of 1513 microns (Figure 3). 


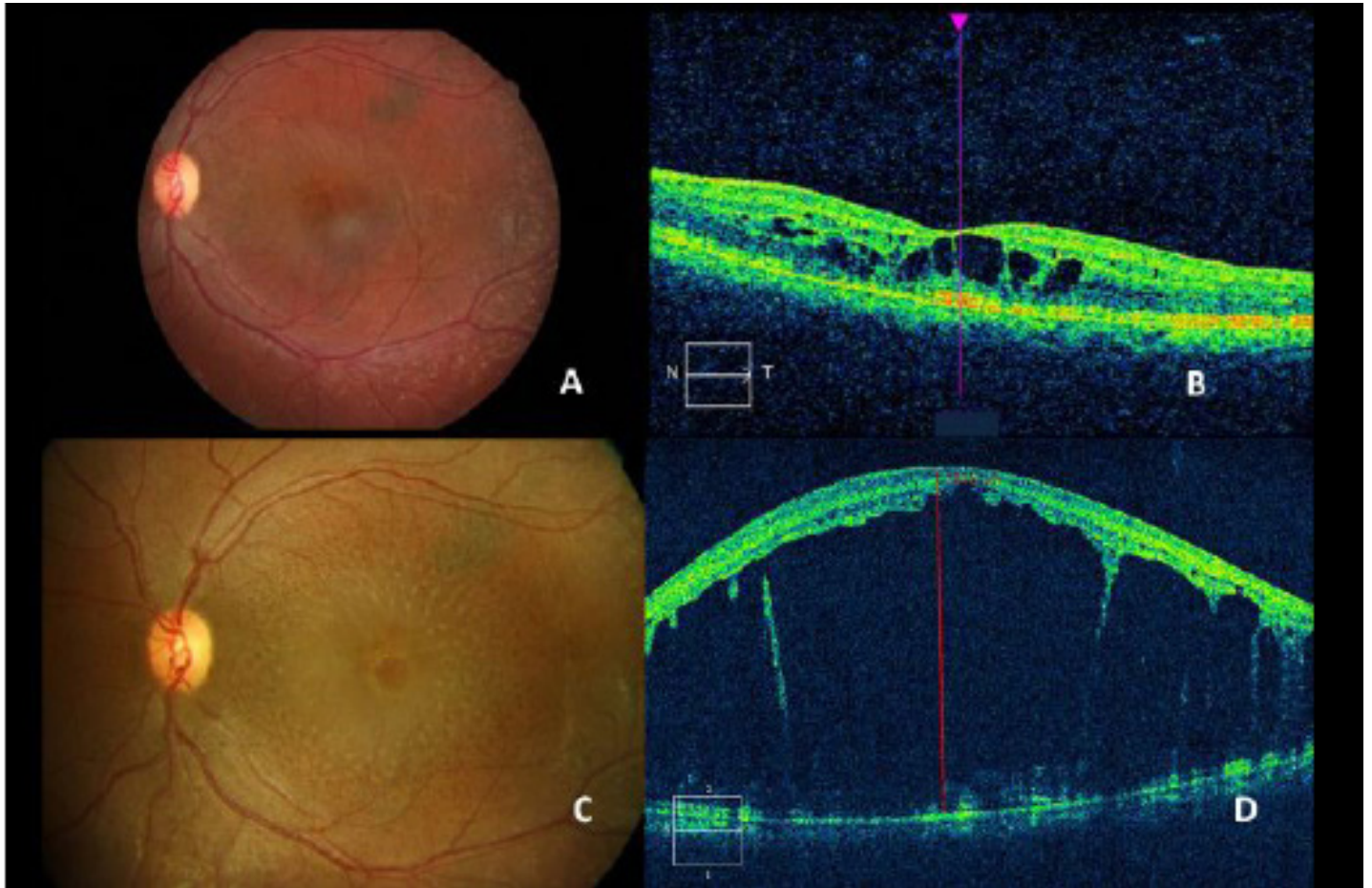

Figure 3: (A-D) showing progression of foveoschesis two years later in the left eye in the color fundus photograph with the characteristic bicycle wheel appearance in the macular area. SD-OCT shows significant elevation of the inner retinal leaflet and confluent macular cystoid spaces

Eager after the intervention for her right eye, the patient consented to a vitrectomy with ILM peel for her left eye, which was followed again by flattening of the central retinoschisis, with complete resolution of the edema in the left eye (Figure 4). VA improved to 4/60 with more notable subjective improvement of visual function than noted on Snellen BCVA. Follow up exams over the next 3 months showed a stable condition with a clear subjective sense of visual improvement that surpassed the increase in visual acuity. Our findings indicate that vitrectomy in this patient had markedly improved macular thickness, BCVA, and subjective visual performance.

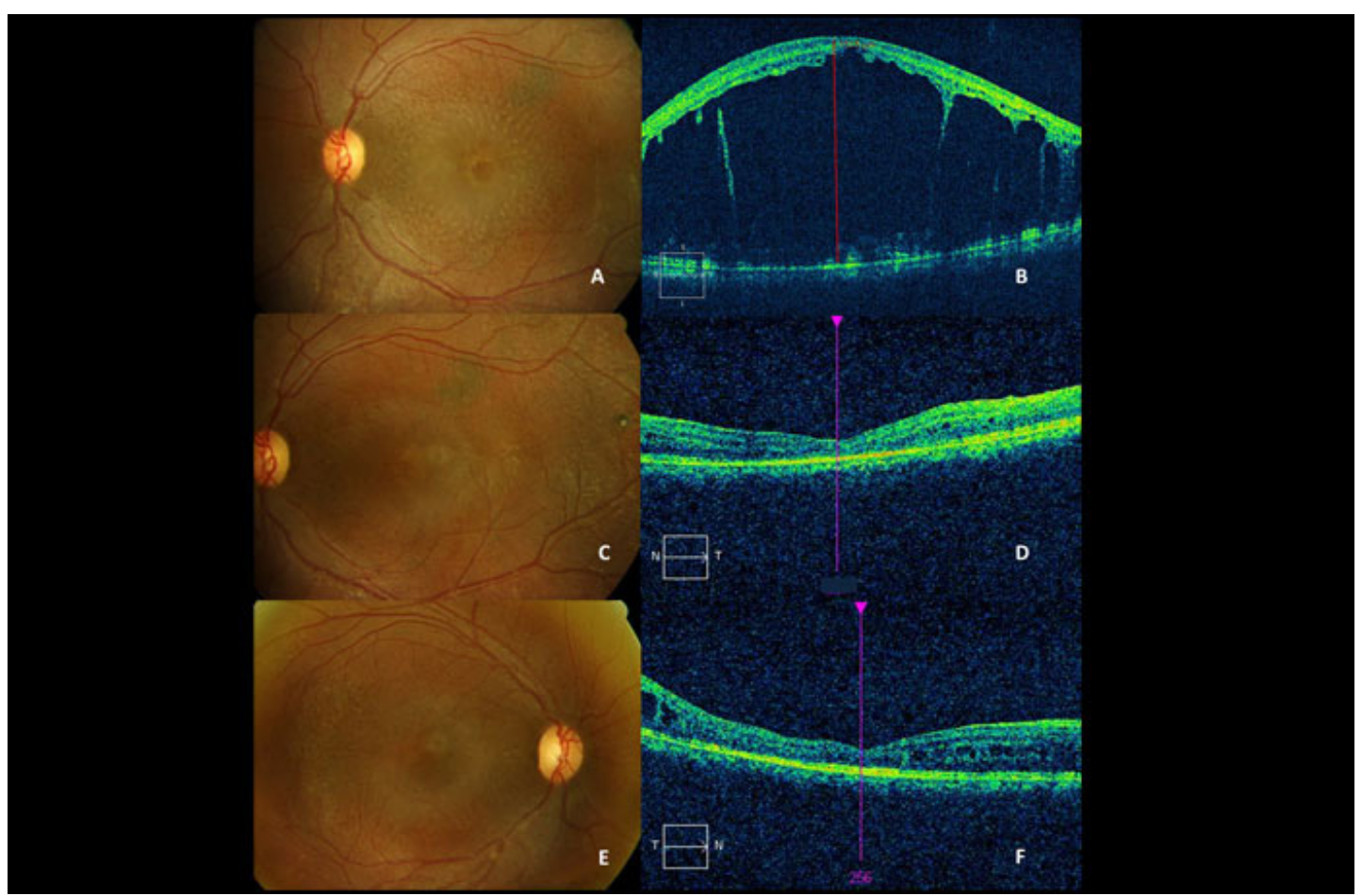

Figure 4: (A-D) demonstrate dramatic improvement in macular thickness, resolution of macular cystoid changes, and flattening of the retinoshesis in the color fundus photo and SD-OCT scans of the left eye three months after vitrectomy with ILM peel; (E and F) document a stable flattened foveoschesis in the right eye two years following surgery 


\section{Discussion}

Goldmann-Favre syndrome, or Enhanced S cone syndrome, is a rare hereditary vitreoretinal degeneration characterized by impaired visual acuity, nyctalopia, vitreous degeneration, peripheral pigmentary anomalies, peripheral and macular retinoschisis with microcystic changes $[1,2]$.

Reports by Kiszkielis [3], Genead [4], and others [5] have demonstrated the efficacy of acetazolamide in some patients with mild cystoid changes, and one by Khairallah et al. [6] on the use of argon laser successfully in one other patient with elevated macular retinoschisis.

The findings after vitrectomy in one, then the other eye of this patient indicate its role in improving BCVA, regression of the macular edema, flattening of the retinoschisis in SD-OCT, and improved subjective as well as objective visual performance.

We do acknowledge the limitation of using a single case report to determine efficacy of certain treatment options and the potential inherent risks with vitreous surgery in this setting. But this report at least documents the favorable outcome with this technique in the patient described when no treatment option is universally effective still for patients with hereditary maculopathies [3-6].

\section{Conclusion}

Despite the inherent risks of surgery, vitrectomy with ILM peel may provide a possible surgical treatment option for patients with elevated retinal foveoschisis associated with enhanced S-cone syndrome.

\section{References}

1. Audo I, Michaelides M, Robson AG, Hawlina M, Vaclavik V, et al. (2008) Phenotypic variation in enhanced S-cone syndrome. Invest Ophthalmol Vis Sci 49: 2082-93.

2. Yzer S, Barbazetto I, Allikmets R, van Schooneveld MJ, Bergen A, et al. (2013) Expanded Clinical Spectrum of Enhanced S-Cone Syndrome. JAMA Ophthalmol 131: 1324-30.

3. Kiszkielis M, Lubiński W, Penkala K (2013) Topical dorzolamide treatment of macular cysts in the enhanced S-cone syndrome patient. Doc Ophthalmol 126: 241-6.

4. Genead MA, Fishman GA, McAnany JJ (2010) Efficacy of topical dorzolamide for treatment of cystic macular lesions in a patient with enhanced S-cone syndrome. Doc Ophthalmol 121: 231-40.

5. Iannaccone A, Fung KH, Eyestone ME, Stone EM (2009) Treatment of Adult-Onset Acute Macular Retinoschisis in Enhanced-S Cone Syndrome with Oral Acetazolamide. Am J Ophthalmol 147: 307-12.

6. Khairallah M, Ladjimi A, Ben Yahia S, Zaouali S, Messaoud R, et al. (2002) Elevated macular retinoschisis associated with Goldmann-Favre syndrome successfully treated with grid laser photocoagulation. Retina 22: 234-7.

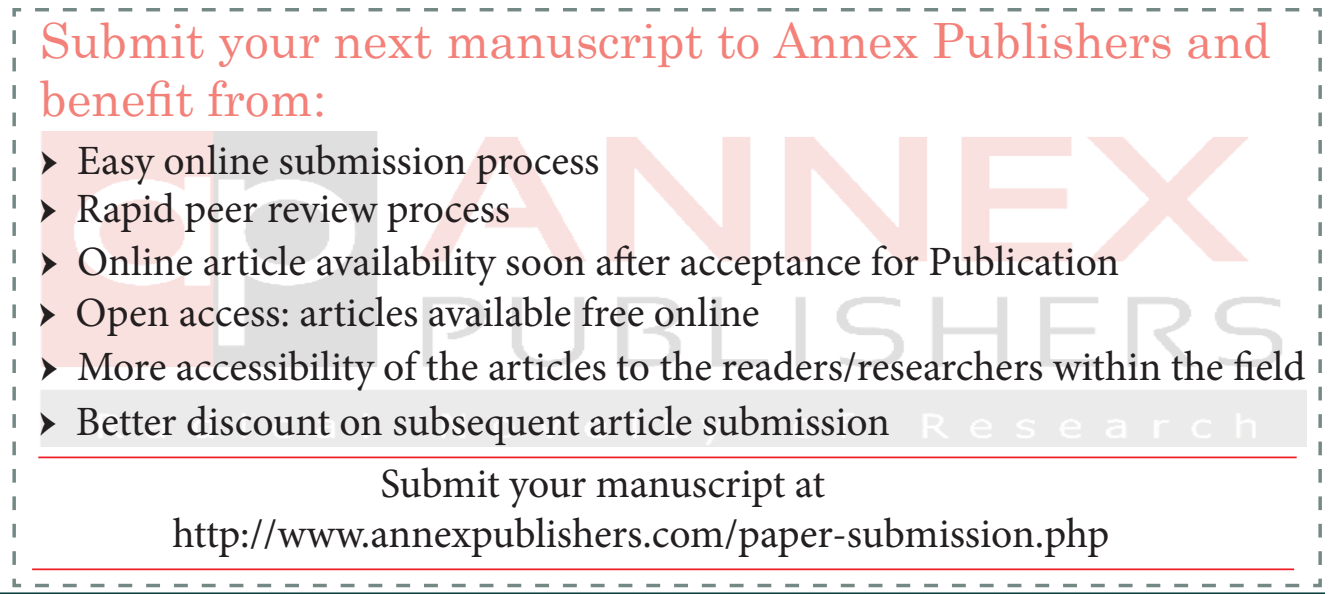

